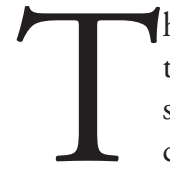
This issue of Healthcare Quarterly includes instructive research results, statistical analyses and casestudy insights within and without the COVID-19 context. It also strikes a highly humanistic tone, a reminder that relationships are at the core of healthcare and that providing and receiving healthcare are profoundly personal and consequential. Organizing, running and resourcing healthcare require strong interpersonal and collective connections, shared accountabilities and agreement on goals that aim for a coherent, continuously improving health system and a safe, fair and caring place to work and heal.

\section{Responding to the COVID-19 Pandemic}

Snowdon and Saunders (2021) cover complex ground in their examination of the impact of the pandemic and the (damaging) interplay between the pandemic, workforce autonomy and the capacity of Canada's health supply chain. The authors draw on and depict hard facts: experiences, conditions and decisions that evoked a medley of emotions and responses from healthcare workers, patients and caregivers, health and union leaders and more. The reflections express personal, professional and "psychological" harm - and even a diagnosis of "mass trauma." All illuminate system, policy and practice issues.

Canada's fragile health supply chain was swamped by the soaring demands for personal protective equipment (PPE) brought on by the COVID-19 pandemic. That has set off a series of unfortunate (and unintended) events leading to the iconic loss of professional autonomy for front-line healthcare workers. Already full of "anticipated fear and uncertainty" about personal safety and infection spread, the workforce was further distressed - and destabilized - when PPE allocation, conservation and rationing began. Healthcare workers could no longer use their expert assessment to determine PPE use; their ethical and professional judgment was superseded by command and control.

Walker et al. (2021) describe a unique interdisciplinary effort by The Ottawa Hospital to prepare non-nursing and non-physician health professionals to safely and effectively work in different disciplines to help manage COVID-19 challenges. Between March and December 2020, approximately 30 health professionals from spiritual care, psychology and social work were redeployed (around 5\% of regulated health professionals). They screened for COVID-19 symptoms in in-patient units and at front entrances, liaised between staff in COVID-19 and non-COVID-19 units and provided personal support at long-term care homes.

The COVID-19 pandemic has caused overwhelming harm, but it has also generated positive results. Guo et al. (2021) share successes in physician engagement - a challenging area to activate at any time - in case studies spanning acute care, chronic care and primary care. Physicians engaged in new activities - all tied to demonstratable quality and systems improvement, such as changing methods to increase vaccinations, introducing a process to address physicianidentified safety issues and adopting mindfulness techniques to tackle burnout.

A chief nursing executive and a physician-in-chief (Strudwick and Stergiopoulos 2021) call for strategic solidarity and a strong, unified and cross-disciplinary voice at mental health leadership tables - an approach they say will secure solutions for everyone and serve the interests of patients and staff. They identify issues requiring "lockstep": dire health human resource challenges, round-the-clock coverage and support for an exhausted and often "burnt out" mental health workforce.

\section{Quality Improvement}

An engaging piece full of robust, relevant data and telling examples by Krantz et al. (2021) highlights the hardships of a highly underserved group facing multiple challenges - parents of children with medical complexity (CMC). It describes how the Navigator Program is helping with emotional, social and economic support.

The program was co-created by community providers and parents of CMC - a core strength. It is supported by a unique healthcare collaboration, including CHEO and PinecrestQueensway Community Health Centre in Ottawa, ON. Parents with lived experience have been part of the program since its inception more than five years ago. They helped shape services and define positions and are program ambassadors and ongoing advisors. Parents and system and knowledge navigators deliver the program, which is also connected to clinical health teams. Rigorous evaluations, including patient experience surveys every six months, ensure that the program meets families' needs. In the early days, the prevalence of medical traumatic stress among families was not fully understood, so the program strengthened its knowledge and clinical capacity to provide better support.

Corkey and Holland (2021) open their article on an empathetic note: "Imagine a patient transferred alone from their well-known community hospital ... left alone on a hard stretcher in a hallway...” (p. 47). That patient experience, identified as a common occurrence with high risk, prompted a four-hospital site in southeastern Ontario to embark on a failure mode and effects analysis (FMEA). That process identified and quantified areas of risk related to interfacility transfers and helped prioritize process and procedural changes. 
Fifty people participated, representing each point of contact or information exchange: emergency department nurses across sites, physicians, physician consultants, staff from the intensive care unit, staff from diagnostic imaging, emergency medical services, the non-urgent patient transport service, bed traffic control and hospitality services. They had never been in the same room before.

FMEA found that "patient preparation" carried $42 \%$ of the overall risk, and patient transfers were largely for diagnostic services. Eliminating that need was a priority addressed by increasing physician consultations through virtual care. The absence of a most responsible provider during all stages of a patient's transfer was rife with risk, so physicians at all sites agreed to practice changes - and increased their appreciation of patient exposure to potential risk.

FMEA produced successful, sustainable strategies for a problem that had long bedevilled a four-hospital organization in southeastern Ontario. Instead of four different patient transfer checklists, there is now one that is shared and streamlined. Because of nurse-physician collaboration, a standard transfer order set will reduce risks from treatment delays. Paramedic service use for non-urgent patient transfers is dramatically reduced. FMEA demanded and delivered compromise. Individuals and teams adjusted historical ideals, dropped the "this is how we always do it here" attitude and embraced change for a common goal: a safe and efficient transfer for every patient.

\section{Workforce Engagement}

Smart and O'Gorman (2021) view workforce engagement as the proverbial glue in a model of care used to good effect by a London-based outpatient orthopaedics physiotherapy team. Elements of the value-based patient-centred care model include safety, quality and patient experience. The authors highlight related achievements: an education program designed by frontline physiotherapists and surgeons to prepare patients for joint replacement surgeries, two-week turnaround to ensure seamless care for patients throughout the COVID-19 pandemic and research on patient and therapist virtual care experiences. The team asserts that these and other examples (highly evaluated interactive events initiated and led by practitioners, resiliency through difficult change and multidisciplinary partnerships) reflect and rely on workplace engagement. The authors say that this glue - with the help of management collaboration - also engenders and augments clinician initiative, leadership and an attitude of joy cultivated by the team's caring culture.

\section{Quarterly Columns}

The ICES report by Duchen et al. (2021) is essential (and encouraging) reading for anyone concerned about Ontario's capacity to marshal health data for improvements in public health. The authors present the role of ICES as a "key component of a resilient information infrastructure" (Duchen et al. 2021: 7), highlighting seminal contributions to producing and enabling health data and revealing difficult truths about neighbourhood-level COVID-19 infection rates and vaccination uptake. From the Canadian Institute for Health Information, Dudevich and Frood (2021) compare data on health system use for March-June 2020 with data from 2019, providing an early analysis of the impact of the COVID-19 pandemic. Twin themes - and an unknown - emerge: sweeping, swift change and a decline across monitored services occurred, along with moves to virtual care, and patients changed how, whether and when they accessed care. What will the impact of delayed or deferred care be?

\section{- The Editors}

\section{References}

Corkey, S. and T. Holland. 2021. Utilizing the Failure Mode and Effects Analysis Tool to Assess and Address Risks Associated with Transitions in Care. Healthcare Quarterly 24(2): 47-52. doi:10.12927/ hcq.2021.26546.

Duchen, R., C. Iskander, H. Chung, J.M. Paterson, J.C. Kwong, S.E. Bronskill et al. 2021. The Role of a Resilient Information Infrastructure in COVID-19 Vaccine Uptake in Ontario. Healthcare Quarterly 24(2): 7-11. doi:10.12927/hcq.2021.26553.

Dudevich, A. and J. Frood. 2021. Impact of the COVID-19 Pandemic on Health System Use in Canada. Healthcare Quarterly 24(2): 12-14. doi:10.12927/hcq.2021.26552.

Guo, M., R. Dunbar-Yaffe, E. Bearss, S. Lim-Reinders and C. Soong. 2021. Do Not Waste a Crisis: Physician Engagement during the COVID-19 Pandemic. Healthcare Quarterly 24(2): 33-37. doi:10.12927/hcq.2021.26549.

Krantz, C., M. Hynes, A. DesLauriers, L.L. Kitcher, T. MacMillan, D. Paradis et al. 2021. Helping Families Thrive: Co-Designing a Program to Support Parents of Children with Medical Complexity. 2021. Healthcare Quarterly 24(2): 40-46. doi:10.12927/hcq.2021.26547.

Smart, M. and P. O'Gorman. 2021. The Relationship between ValueBased Care Workforce Engagement and Clinical Leadership: Learning from an Outpatient Physiotherapy Team. Healthcare Quarterly 24(2): 53-58. doi:10.12927/hcq.2021.26545.

Snowdon, A.W. and M. Saunders. 2021. COVID-19, Workforce Autonomy and the Health Supply Chain. Healthcare Quarterly 24(2): 15-26. doi:10.12927/hcq.2021.26551.

Strudwick, G. and V. Stergiopoulos. 2021. Mental Health Clinician Leaders in "Lockstep" as a Necessary Means to Address Care Challenges during the Pandemic. Healthcare Quarterly 24(2): 38-39. doi:10.12927/hcq.2021.26548.

Walker, L.A.S., A.J. Pontefract and D.A. Bournes. 2021. Health Professional Redeployment and Cross-Training in Response to the COVID-19 Pandemic. Healthcare Quarterly 24(2): 27-32. doi:10.12927/hcq.2021.26550. 